\title{
Solution for static, spherically symmetric Lovelock gravity coupled with Yang-Mills hierarchy
}

\author{
S. Habib Mazharimousavi* , M. Halilsoy \\ Department of Physics, Eastern Mediterranean University, G. Magusa, North Cyprus, Mersin 10, Turkey
}

\section{A R T I C L E I N F O}

\section{Article history:}

Received 28 July 2010

Received in revised form 1 September 2010

Accepted 15 September 2010

Available online 18 September 2010

Editor: A. Ringwald

\section{Keywords:}

Black-holes

Lovelock gravity

\begin{abstract}
A B S T R A C T
The hierarchies of both Lovelock gravity and power-Yang-Mills field are combined through gravity in a single theory. In static, spherically symmetric ansatz exact particular integrals are obtained in all higher dimensions. The advantage of such hierarchies is the possibility of choosing coefficients, which are arbitrary otherwise, to cast solutions into tractable forms. To our knowledge the solutions constitute the most general spherically symmetric metrics that incorporate complexities both of Lovelock and YangMills hierarchies within the common context. A large portion of our general class of solutions concerns and addresses to black holes for which specific examples are given. Thermodynamical behaviors of the system is briefly discussed in particular dimensions.
\end{abstract}

(C) 2010 Elsevier B.V. All rights reserved.

\section{Introduction}

The hierarchy of Lovelock gravity consists of a $\operatorname{sum}\left(\sum_{s=0} \alpha_{s} L_{s}\right.$, $\alpha_{s}=$ constant, $L_{s}=$ sth order Lagrangian) of geometrical terms representing higher corrections in suitable combinations that do not give rise to equations higher than second order [1]. The higher order terms are reminiscent of higher order Feynman diagrams in field theory but all at a classical level. The zeroth order term $(s=0)$ in the hierarchy is simply the cosmological term while the first order $(s=1)$ one corresponds to the familiar Einstein-Hilbert (EH) Lagrangian. The second order $(s=2)$ term gives the GaussBonnet (GB) gravity with the quadratic invariants. The third and higher order Lovelock terms grow rather wildly, giving the impression that it is impossible to keep the track analytically. Contrary to the expectations, however, in particular geometries exact solutions are available to all orders of the hierarchy. Not only the geometric terms but with various sources, including power-Maxwell and power-Yang-Mills (YM) fields, exact solutions are available in static, spherical symmetric ansatz [2,3]. By the power-Maxwell/YM, it is implied that the invariants in the Lagrangian are raised to a power $k$. The finely-tuned power has physical implication as far as energy conditions are concerned [3]. In principle, $k$ can be chosen as an arbitrary $( \pm)$ rational number, but such a freedom raises problems when the energy and causality conditions are imposed. (Based on the energy conditions, $k$ must be at least greater than $\frac{1}{2}$. Here in our study, since we aimed to consider a discrete hierarchy,

\footnotetext{
* Corresponding author.

E-mail addresses: habib.mazhari@emu.edu.tr (S.H. Mazharimousavi), mustafa.halilsoy@emu.edu.tr (M. Halilsoy).
}

we restrict ourselves to the integer $k$ although this is not the only possible choice. In other words one may consider a continuous hierarchy with $\frac{1}{2}<k \in \mathbb{R}$ which may be studied separately.) For this reason, to be on the safe side we choose $k=(+)$ integer in this study. The topological implication of such powers, if there is any at all, remains to be seen.

In this Letter, coupled with the Lovelock hierarchy we consider the YM hierarchy (a different approach to YM hierarchy was first introduced by D.H. Tchrakian in 1985 [4] and the concept was expanded later [5]) of the form $\sim \sum_{k} b_{k} \mathcal{F}^{k}$ where $b_{k}$ are constant coefficients and $\mathcal{F}=$ the YM invariant $=F_{\mu \nu}^{(a)} F^{(a) \mu \nu}$, with the internal index $a$.

It is interesting to note that for the YM invariant and dimension of spacetime $d>5, \mathcal{F} \sim \frac{1}{r^{4}}$, irrespective of the dimension. In the Maxwell case we recall that the invariant $\mathcal{F}_{M} \sim \frac{1}{r^{2(d-2)}}$, depends on the dimension as well. (The reason that we excluded $d=5$ in the YM case is that it contains a logarithmic term and violates the rule as aforesaid [6].) This suggests, as a matter of fact, that we have a working YM hierarchy whereas for the Maxwell case a similar hierarchy does not work with equal ease. In obtaining an exact integral to the problem we make use of a theorem proved beforehand which is valid for a large class of energy-momenta [7]. Here, in particular we evaluate the integral for the general YM field arising from the Wu-Yang ansatz [8]. Let us add that it is this particular ansatz which makes the YM hierarchy tractable in a diagonal metric, simply by making the YM invariant mentioned above to have a fixed power. It should be supplemented that the $\mathrm{Wu}$-Yang ansatz in our choice works only for the pure magnetic YM fields. Any other YM ansatz that can be extended to higher dimensions analytically, even with a power (and hierarchy), 
remains to be seen. The energy and causality conditions which are employed in Appendix A determine the acceptable integers as a function of dimensionality in our solution. These split naturally into two broad classes labelled by 'even' and 'odd'. The intricate structure of our solutions dashes hopes to determine horizons and thermodynamical functions analytically. In principle, however, we obtain infinite class of solutions pertaining to all dimensions that incorporate Lovelock and YM hierarchies in the common metric. We choose particular parameters and dimensions to present working examples of black hole solutions which elucidate our general class. The 5-dimensional black hole solution with an effective mass defined from cosmological constant and YM charge is one such example. Chern-Simons (CS) black hole solution in $d=11$ constitutes another example as an application of our general class. From the definition of specific heat we show the absence of thermodynamical phase transition for the CS black hole in $d=11$.

\section{2. d-dimensional Einstein-Lovelock gravity with YM hierarchy}

The $d$-dimensional action for Einstein-Lovelock-Yang-Mills hierarchies with a cosmological constant $\Lambda$ is given by $(8 \pi G=1)$

$I=\frac{1}{2} \int d x^{d} \sqrt{-g}\left(\sum_{s=0}^{\left[\frac{d-1}{2}\right]} \alpha_{s} L_{s}-\sum_{k}^{q} b_{k} \mathcal{F}^{k}\right)$

in which $\alpha_{0}=-\frac{(d-2)(d-1)}{3} \Lambda, \alpha_{1}=1, \mathcal{F}$ is the YM invariant

$\mathcal{F}=\boldsymbol{\gamma}_{a b}\left(F_{\mu \nu}^{(a)} F^{(b) \mu \nu}\right)$,

$a, b=1,2, \ldots, \frac{(d-2)(d-1)}{2}$ and $\gamma_{a b}=\delta_{a b}$.

The parameter $q(1 \leqslant k \leqslant q)$ is an integer, $\alpha_{s}$ stand for arbitrary constants, $\left[\frac{d-1}{2}\right]$ represents the integer part, and the Lovelock Lagrangian is

$L_{s}=2^{-n} \delta_{c_{1} d_{1} \ldots c_{n} d_{n}}^{a_{1} b_{1} \ldots a_{n} b_{n}} R^{c_{1} d_{1}}{ }_{a_{1} b_{1}} \ldots R^{c_{s} d_{s}}{ }_{a_{s} b_{s}}, \quad s \geqslant 1$.

Variation with respect to the gauge potentials $\mathbf{A}^{(a)}$ yields the YM equations

$\sum_{k} b_{k}\left\{\mathbf{d}\left({ }^{\star} \mathbf{F}^{(a)} \mathcal{F}^{k-1}\right)+\frac{1}{\sigma} C_{(b)(c)}^{(a)} \mathcal{F}^{k-1} \mathbf{A}^{(b)} \wedge{ }^{\star} \mathbf{F}^{(c)}\right\}=0$,

where * means duality, $C_{(b)(c)}^{(a)}$ stands for the structure constants of $\frac{(d-2)(d-1)}{2}$-parameter Lie group $G, \sigma$ is a coupling constant and $\mathbf{A}^{(a)}$ are the $S O(d-1)$ gauge YM potentials. The determination of the components $C_{(b)(c)}^{(a)}$ has been described elsewhere [9]. We note that the internal indices $\{a, b, c, \ldots\}$ do not differ whether in covariant or contravariant form. Variation of the action with respect to the spacetime metric $g_{\mu \nu}$ yields the field equations

$\sum_{s=0}^{\left[\frac{d-1}{2}\right]} \alpha_{s} G_{\mu}^{v(s)}=T_{\mu}^{v}$

where

$T^{\mu}{ }_{\nu}=-\frac{1}{2} \sum_{k} b_{k}\left(\delta^{\mu}{ }_{\nu} \mathcal{F}^{k}-4 k \gamma_{a b}\left(F_{\nu \lambda}^{(a)} F^{(b) \mu \lambda}\right) \mathcal{F}^{k-1}\right)$,

is the energy-momentum tensor representing the matter fields, and

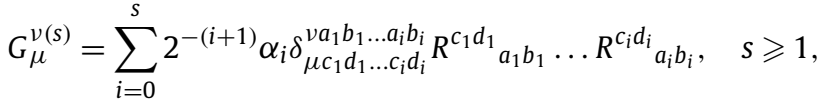

$G_{\mu}^{\nu(0)}=\frac{(d-2)(d-1)}{6} \Lambda \delta_{\mu}^{\nu} \quad(s=0)$.

Our metric ansatz for $d$-dimensions, is chosen as

$d s^{2}=-f(r) d t^{2}+\frac{d r^{2}}{f(r)}+r^{2} d \Omega_{(d-2)}^{2}$,

in which $f(r)$ is our metric function. The choice of these metrics can be traced back to the form of the stress-energy tensor (6), which satisfies $T_{0}^{0}-T_{1}^{1}=0$ (see Eq. (12) below) and consequently $G_{0}^{0}-G_{1}^{1}=0$, whose explicit form, on integration, gives $\left|g_{00} g_{11}\right|=$ $C=$ constant. We need only to choose the time scale at infinity to make this constant equal to unity.

Recently we have introduced and used the higher dimensional version of the Wu-Yang [8] ansatz in EYM theory of gravity [8]. In this ansatz we express the Yang-Mills magnetic gauge potential one-forms in the following manner

$\mathbf{A}^{(a)}=\frac{Q}{r^{2}} C_{(i)(j)}^{(a)} x^{i} d x^{j}, \quad Q=$ YM magnetic charge, $r^{2}=\sum_{i=1}^{d-1} x_{i}^{2}$,

$2 \leqslant j+1 \leqslant i \leqslant d-1$, and $1 \leqslant a \leqslant \frac{(d-2)(d-1)}{2}$,

$x_{1}=r \cos \theta_{d-3} \sin \theta_{d-4} \ldots \sin \theta_{1}$,

$x_{2}=r \sin \theta_{d-3} \sin \theta_{d-4} \ldots \sin \theta_{1}$,

$x_{3}=r \cos \theta_{d-4} \sin \theta_{d-5} \ldots \sin \theta_{1}$,

$x_{4}=r \sin \theta_{d-4} \sin \theta_{d-5} \ldots \sin \theta_{1}$,

$x_{d-1}=r \cos \theta_{1}$.

One can easily show that these ansaetze satisfy the YM equations $[6,8]$. In consequence, the energy-momentum tensor (6), with

$\mathcal{F}=\frac{(d-2)(d-1) Q^{2}}{r^{4}}$,

$\operatorname{Tr}\left(F_{\theta_{i} \lambda}^{(a)} F^{(a) \theta_{i} \lambda}\right)=\frac{(d-3) Q^{2}}{r^{4}}=\frac{1}{d-2} \mathcal{F}$

takes the compact form

$$
\begin{aligned}
& T^{\mu}{ }_{\nu}=-\frac{1}{2} \sum_{k} b_{k} \mathcal{F}^{k} \operatorname{diag}[1,1, \xi, \xi, \ldots, \xi], \\
& \text { with } \xi=\left(1-\frac{4 k}{d-2}\right) .
\end{aligned}
$$

\subsection{Energy conditions and the solutions}

Upon choosing the energy-momentum tensor, it is necessary to look at the energy conditions. This is important, because the upper and lower limits of $k$ will come to light by imposing the energy and causality conditions all satisfied. In a straightforward calculation (see Appendix A) one can show that WEC, SEC, DEC and CC are all satisfied if and only if $\frac{d-1}{4} \leqslant k<\frac{d-1}{2}$. Therefore we should modify our summation symbol accordingly as

$\sum_{k} b_{k} \rightarrow \sum_{k=-\left[-\frac{d-1}{4}\right]}^{-\left[-\frac{d-1}{2}\right]-1} b_{k}$ 
Here one should notice that in 4 and 5 dimensions only $b_{1}$ is available and for 6 and 7 dimensions $b_{2}$ is nonzero. Of course, for $d$-dimensions we have $\left[-\frac{d-1}{4}\right]-\left[-\frac{d-1}{2}\right]$ terms included. Our static, spherically symmetric metric is given by (8), whose metric function can be re-expressed, for convenience in the form

$f(r)=1-r^{2} H(r)$,

and from the $t t$ component of (5) and (12) we obtain [7]

$\sum_{s=0}^{\left[\frac{d-1}{2}\right]} \tilde{\alpha}_{s} H^{s}=\frac{4 m}{(d-2) r^{d-1}}-\frac{2}{(d-2) r^{d-1}} \int r^{d-2} T_{t}^{t} d r$

Here $m$ is an integration constant related to the ADM mass of the black hole, $\tilde{\alpha}_{0}=-\frac{\Lambda}{3}, \tilde{\alpha}_{1}=1$, and

$\tilde{\alpha}_{s}=\prod_{i=3}^{2 s}(d-i) \alpha_{s}, \quad s>1$.

Now, we use $T_{t}^{t}$ given in (12) to get

$$
\begin{aligned}
\sum_{s=0}^{\left[\frac{d-1}{2}\right]} \tilde{\alpha}_{s} H^{s}= & \frac{4 m}{(d-2) r^{d-1}} \\
& +\sum_{k=-\left[-\frac{d-1}{4}\right]}^{-\left[-\frac{d-1}{2}\right]-1} \frac{b_{k} \tilde{Q}_{k}^{2}}{(d-2)}\left\{\begin{array}{ll}
\frac{1}{(d-1-4 k) r^{4 k}}, & k \neq \frac{d-1}{4} \\
\frac{\ln r}{r^{d-1}}, & k=\frac{d-1}{4}
\end{array}=\Psi,\right.
\end{aligned}
$$

where $\tilde{Q}_{k}^{2}=\left((d-2)(d-1) Q^{2}\right)^{k}$ and $\Psi$ abbreviates the indicated series. Here we comment that at $r \rightarrow \infty$, one gets

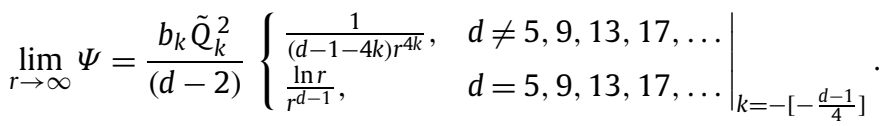

Let's introduce new parameters as

$\tilde{\alpha}_{s}=\frac{\bar{\alpha}_{s}}{\bar{\alpha}_{1}}, \quad$ for $s \geqslant 2$ and $-\frac{\Lambda}{3}=\frac{\bar{\alpha}_{0}}{\bar{\alpha}_{1}}$,

which lead to

$\sum_{s=0}^{\left[\frac{d-1}{2}\right]} \bar{\alpha}_{s} H^{s}=\bar{\alpha}_{1} \Psi$

and choose a specific set of [10] $\bar{\alpha}_{s}$ such that

$\bar{\alpha}_{s}=( \pm 1)^{s+1}\left(\begin{array}{c}{\left[\frac{d-1}{2}\right]} \\ s\end{array}\right) \ell^{2 s-d}$

where $-\frac{\Lambda}{3}=\frac{\bar{\alpha}_{0}}{\bar{\alpha}_{1}}= \pm \frac{\ell^{-2}}{\left[\frac{d-1}{2}\right]}$. Following the latter expression, Eq. (20) gives

$\left(1 \pm \ell^{2} H\right)^{\left[\frac{d-1}{2}\right]}= \pm \ell^{d} \bar{\alpha}_{1} \Psi$

and consequently

$f^{( \pm)}(r)=1 \pm \frac{r^{2}}{\ell^{2}} \mp \frac{r^{2}}{\ell^{2}} \sigma\left( \pm\left[\frac{d-1}{2}\right] \ell^{2} \Psi\right)^{1 /\left[\frac{d-1}{2}\right]}$,

in which

$\sigma= \begin{cases} \pm 1, & {\left[\frac{d-1}{2}\right]=\text { even }} \\ 1, & {\left[\frac{d-1}{2}\right]=\text { odd }}\end{cases}$
After this general solution we label the solutions for even and odd dimensions separately. To do so, we put $\left[\frac{d-1}{2}\right]=\frac{d-1}{2}$ for odd dimensions and $\left[\frac{d-1}{2}\right]=\frac{d-2}{2}$ for even dimensions into (23) to obtain the splitting

$$
\begin{aligned}
f_{\text {even }}^{( \pm)}(r)= & 1 \pm \frac{r^{2}}{\ell^{2}} \mp \sigma\left[ \pm \frac{d-2}{2 \ell^{d-4}}\left(\frac{4 m}{(d-2) r}+\sum_{k=-\left[-\frac{d-1}{4}\right]}^{-\left[-\frac{d-1}{2}\right]-1} \frac{b_{k} \tilde{Q}_{k}^{2}}{(d-2)}\right.\right. \\
& \times \begin{cases}\frac{1}{(d-1-4 k) r^{4 k-d+2}}, & k \neq \frac{d-1}{4} \\
\frac{\ln r}{r}, & \left.\left.k=\frac{d-1}{4}\right)\right]^{\frac{2}{d-2}}\end{cases}
\end{aligned}
$$

and

$$
\begin{aligned}
f_{\text {odd }}^{( \pm)}(r)= & \pm \frac{r^{2}}{\ell^{2}} \mp \sigma\left[ \pm \frac{d-1}{2 \ell^{d-3}}\left(\frac{4 m}{(d-2)}+\sum_{k=-\left[-\frac{d-1}{4}\right]}^{-\left[-\frac{d-1}{2}\right]-1} \frac{b_{k} \tilde{Q}_{k}^{2}}{(d-2)}\right.\right. \\
& \times \begin{cases}\frac{1}{(d-1-4 k) r^{4 k-d+1}}, & k \neq \frac{d-1}{4} \\
\ln r, & \left.\left.k=\frac{d-1}{4}\right)\right]^{\frac{2}{d-1}}\end{cases}
\end{aligned}
$$

From $f_{\text {even }}^{( \pm)}(r)$, for instance the Einstein-de Sitter limit can readily be seen for $d=4$ and $Q_{k}=0$. It is remarkable to observe that by setting $b_{k}=0$ we obtain

$\left.f_{\text {even }}^{( \pm)}(r)\right|_{b_{k}=0}=1 \pm \frac{r^{2}}{\ell^{2}} \mp \sigma\left[ \pm \frac{1}{\ell^{d-4}} \frac{2 m}{r}\right]^{\frac{2}{d-2}}$

and

$\left.f_{\text {odd }}^{( \pm)}(r)\right|_{b_{k}=0}=1 \pm \frac{r^{2}}{\ell^{2}} \mp \sigma\left[ \pm \frac{1}{\ell^{d-3}}\left(\frac{2(d-1) m}{(d-2)}\right)\right]^{\frac{2}{d-1}}$

which by choosing the positive branches and redefinition of the free parameters we get the results reported in [11]. Therefore we use only the positive branches for our further study. Here we investigate the possible horizon of the above black hole solutions.

\subsubsection{Even dimensions}

To find the horizon(s) of the solution given in Eq. (25) we set

$f_{\text {even }}^{(+)}\left(r_{h}\right)=0$

which admits the relation between the black hole's parameters. Finding horizon(s) in a closed form is not possible, therefore we choose a specific dimension, namely $d=8$ for going further. In this setting the latter equation reads

$1+\frac{r_{h}^{2}}{\ell^{2}}-\left[\frac{1}{\ell^{4}}\left(\frac{2 m}{r_{h}}-\frac{b_{2} \tilde{Q}_{2}^{2}}{2} \frac{1}{r_{h}^{2}}-\frac{b_{3} \tilde{Q}_{3}^{2}}{10} \frac{1}{r_{h}^{6}}\right)\right]^{\frac{1}{3}}=0$.

Fig. 1 displays $\rho=\frac{r_{h}}{\ell}$ in terms of $\mu=\frac{b_{2} \tilde{Q}_{2}^{2}}{2 \ell^{6}}, v=\frac{b_{3} \tilde{Q}_{3}^{2}}{10 \ell^{10}}$ for $\frac{m}{\ell^{5}}=1$. Depending on $\mu$ and $v$, two horizons or no horizon cases are the basic information that Fig. 1 reveals. Changing $m$ does not effect the general schema of the figure. From the metric one observes that $r=0$ is a singularity hidden by horizon(s) and the Ricci scalar, once $r \rightarrow 0$ behaves as

$\lim _{r \rightarrow 0} R \rightarrow \frac{-12 v^{1 / 3}}{\ell^{2 / 3} r^{4}} \rightarrow \pm \infty$. 


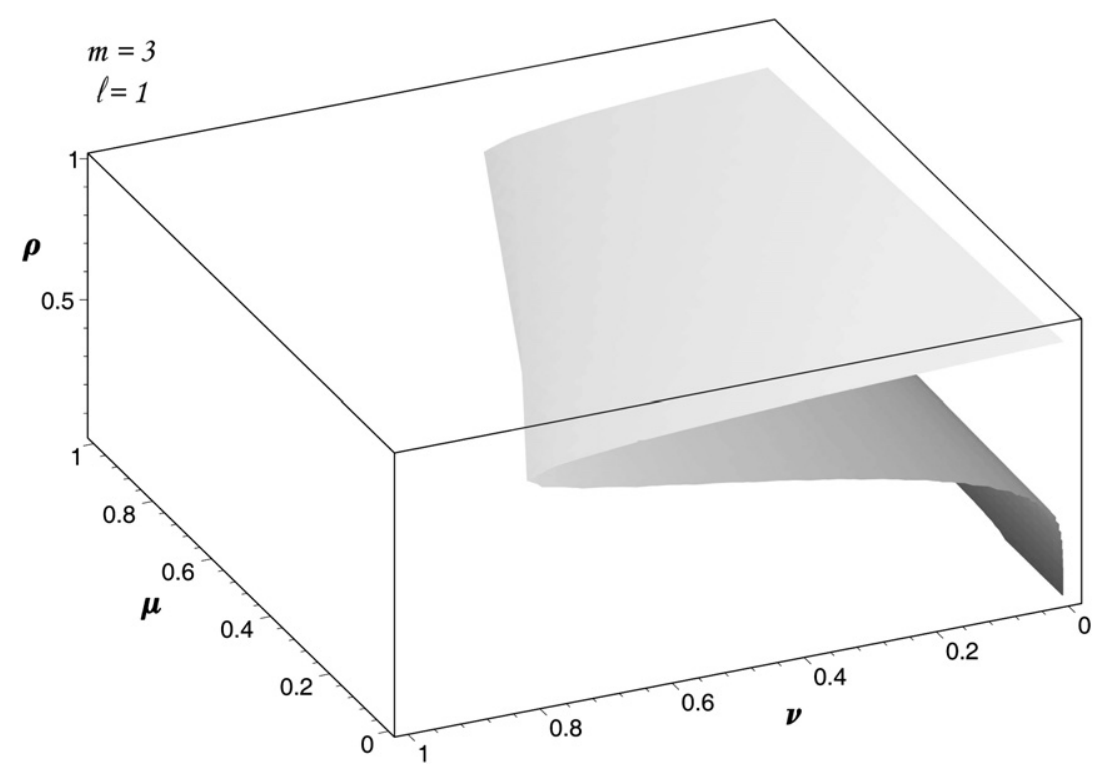

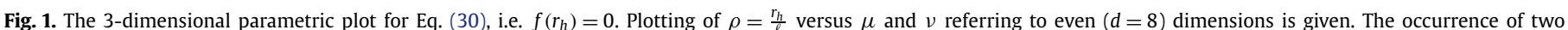

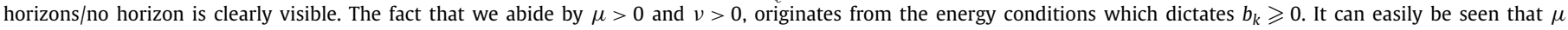
plays little role in comparison with $v$.

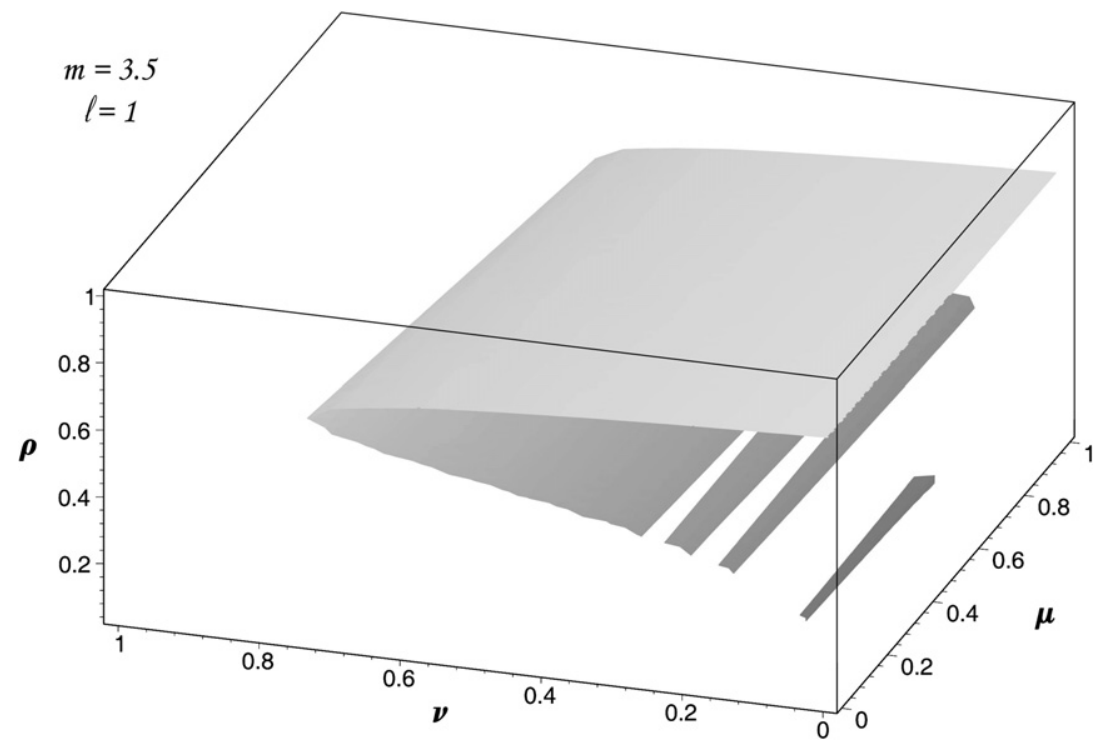

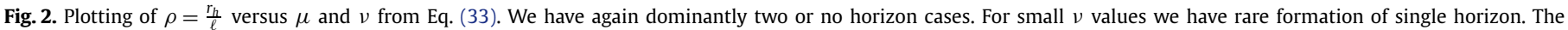
effect of $v$ dominates over $\mu$ also here for odd $(d=9)$ dimensions.

\subsubsection{Odd dimensions}

Again, in this part, we set the metric function (26) to zero, i.e.,

$f_{\text {odd }}^{(+)}\left(r_{h}\right)=0$

which after we choose a specific odd dimension, namely $d=9$ it reads

$1+\frac{r_{h}^{2}}{\ell^{2}}-\sigma\left[\frac{1}{\ell^{6}}\left(\frac{16 m}{7}+\frac{4 b_{2} \tilde{Q}_{2}^{2}}{7} \ln r_{h}-\frac{b_{3} \tilde{Q}_{3}^{2}}{7} \frac{1}{r_{h}^{4}}\right)\right]^{\frac{1}{4}}=0$

Unlike the previous example, here $\sigma= \pm 1$ but for $\sigma=-1$ definitely there is no horizon and our solution collapses to a cosmological object which is not of interest. For $\sigma=+1$ the solution admits black hole with horizon(s). In Fig. 2 we plot $\rho=\frac{r_{h}}{\ell}$ in terms of $\mu=\frac{4 b_{2} \tilde{Q}_{2}^{2}}{7 \ell^{6}}, v=\frac{b_{3} \tilde{Q}_{3}^{2}}{7 \ell^{10}}$ and for $\frac{16 m}{7 \ell^{6}}=\mu \frac{\ln \ell^{2}}{\ell^{6}}+1$. We observe that Fig. 2 shares much of the features with Fig. 1. One should notice that in this case we have the condition

$\frac{1}{\ell^{6}}\left(\frac{16 m}{7}+\frac{4 b_{2} \tilde{Q}_{2}^{2}}{7} \ln r_{h}-\frac{b_{3} \tilde{Q}_{3}^{2}}{7} \frac{1}{r_{h}^{4}}\right) \geqslant 0$.

Although these two examples are given in specific dimensions, they show how the procedure goes on and definitely in higher dimensions having more terms in the hierarchy makes the analysis much more complicated. Let us add that the Ricci scalar in this case diverges as $r^{-3}$ which shows that $r=0$ is a singular point hidden behind the horizon(s). 


\subsection{A very specific case}

Now let us relax the energy conditions except the WEC which allows us to choose $k=0,1, \ldots,\left[\frac{d-1}{2}\right]$. For the case of $k \neq \frac{d-1}{4}$ one finds from (17) that

$\sum_{s=0}^{\left[\frac{d-1}{2}\right]} \bar{\alpha}_{s} H^{s}=\bar{\alpha}_{1}\left(\frac{4 m}{(d-2) r^{d-1}}+\sum_{k=0}^{\left[\frac{d-1}{2}\right]} b_{k} \frac{\tilde{Q}_{k}^{2}}{(d-2)(d-1-4 k) r^{4 k}}\right)$

which after setting $m=0$ and $\frac{b_{k} \tilde{Q}_{k}^{2}}{(d-2)(d-1-4 k)}=\beta_{k}=( \pm 1)^{k+1} \times$ $\left(\begin{array}{c}\left.\frac{d-1}{2}\right] \\ k\end{array}\right) \lambda^{2 k-d}$ this admits

$\sum_{s=0}^{\left[\frac{d-1}{2}\right]} \bar{\alpha}_{s} H^{s}=\bar{\alpha}_{1} \sum_{k=0}^{\left[\frac{d-1}{2}\right]} \beta_{k}\left(\frac{1}{r^{4}}\right)^{k}$

This yields

$\sum_{s=0}^{\left[\frac{d-1}{2}\right]}( \pm 1)^{s}\left(\begin{array}{c}{\left[\frac{d-1}{2}\right]} \\ s\end{array}\right) \ell^{2 s-d} H^{s}=\sum_{k=0}^{\left[\frac{d-1}{2}\right]}( \pm 1)^{k}\left(\begin{array}{c}{\left[\frac{d-1}{2}\right]} \\ k\end{array}\right) \lambda^{2 k-d}\left(\frac{1}{r^{4}}\right)^{k}$

or

$\ell^{-d}\left(1 \pm \ell^{2} H\right)^{\left[\frac{d-1}{2}\right]}=\bar{\alpha}_{1} \lambda^{-d}\left(1 \pm \frac{\lambda^{2}}{r^{4}}\right)^{\left[\frac{d-1}{2}\right]}$

which, after adjusting $\bar{\alpha}_{1} \lambda^{-d}=\ell^{-d}$ one finds

$\left(1 \pm \ell^{2} H\right)^{\left[\frac{d-1}{2}\right]}=\left(1 \pm \frac{\lambda^{2}}{r^{4}}\right)^{\left[\frac{d-1}{2}\right]}$

and depending on the dimensionality we have

$1 \pm \ell^{2} H=\sigma\left(1 \pm \frac{\lambda^{2}}{r^{4}}\right)$

This leads to

$H=\mp \frac{1}{\ell^{2}} \pm \frac{\sigma}{\ell^{2}}\left(1 \pm \frac{\lambda^{2}}{r^{4}}\right)$

and consequently

$$
\begin{aligned}
f(r)= & 1 \pm \frac{r^{2}}{\ell^{2}} \mp \frac{\sigma}{\ell^{2}}\left(r^{2} \pm \frac{\lambda^{2}}{r^{2}}\right) \\
= & \left\{\begin{array}{c}
1-\frac{\lambda^{2}}{\ell^{2}} \frac{1}{r^{2}} \\
d=7,8,11,12,15,16, \ldots \\
1-\frac{\lambda^{2}}{\ell^{2}} \frac{1}{r^{2}} \text { and } 1 \pm \frac{2}{\ell^{2}} r^{2}+\frac{\lambda^{2}}{\ell^{2}} \frac{1}{r^{2}} \\
d=5,6,9,10,13,14, \ldots
\end{array}\right.
\end{aligned}
$$

It is remarkable to observe that the latter solution is nothing but the Schwarzschild black hole-like solution in 5-dimensions if we consider $\frac{\lambda^{2}}{\ell^{2}}$ as the effective mass of the black hole. Note that the mass term of the black hole, $m$ was chosen to be zero. Also, for the other set of solutions i.e.

$f(r)=1 \pm \frac{2}{\ell^{2}} r^{2}+\frac{\lambda^{2}}{\ell^{2}} \frac{1}{r^{2}}$

one may call it anti-Schwarzschild black hole with a positive or negative cosmological constant. To get a better idea about this solution we rewrite it in terms of $m_{e f f}=\frac{\lambda^{2}}{\ell^{2}}$ and $\Lambda_{\text {eff }}= \pm \frac{2}{\ell^{2}}$, so that
$f(r)=1+\Lambda_{e f f} r^{2}+\frac{m_{e f f}}{r^{2}}$.

Let us remind, from the above identifications, that $m_{\text {eff }}$ depends on both $\ell$ and $Q_{k}$. It is clear that with positive sign there is no horizon and therefore it is a cosmological object which has a naked singularity at the origin. The negative branch has a cosmological horizon at

$r_{h}=\left(\frac{\ell^{2}+\sqrt{\ell^{2}+8 \lambda^{2}}}{4}\right)^{1 / 2}$.

\subsection{Example of Chern-Simons (CS) gravity in 11-dimensions}

As one may notice, setting the $\left[\frac{d-1}{2}\right]$ Lovelock parameters according to (21), in odd dimensions it becomes isometric with the CS theory of gravity [10-12]. Therefore Eq. (26) gives a black hole solution in CS theory, and in this section we shall go through some of the physical properties of this type BHs in 11-dimensions as an example.

\subsubsection{For $k \neq \frac{d-1}{4}$ with positive branch}

The solution, after choosing $\ell^{-2}=-\left[\frac{d-1}{2}\right] \frac{\Lambda}{3}=1$ and rewriting the integration constants, in 11-dimensions, reads

$f_{\text {odd }}(r)=1+r^{2}-\left(1+M-\frac{\mu}{r^{2}}-\frac{v}{r^{6}}\right)^{\frac{1}{5}}$

in which $\mu=202500 b_{3} Q^{6}, v=6075000 b_{4} Q^{8}$ and $M=\frac{20}{9} m$. We remark that although the constants $\mu$ and $v$ are multipole-like coefficients depending on powers of the YM charge $Q$ and cosmological constant, which is scaled to unity, their exact interpretation can be understood upon expansion of the power. From the energy conditions (see Appendix A) we show that $b_{k} \geqslant 0$; this implies restriction on the mass parameter $M$ so that the parenthesis in (45) is positive. The Hawking temperature and the mass of the black hole are given by

$T_{H}=\frac{1}{4 \pi} f^{\prime}\left(r_{h}\right)=\frac{r_{h}}{2 \pi}-\frac{\mu}{10 \pi r_{h}^{3}\left(1+r_{h}^{2}\right)^{4}}-\frac{3 v}{10 \pi r_{h}^{7}\left(1+r_{h}^{2}\right)^{4}}$,

and

$M=\frac{\mu}{r_{h}^{2}}+\frac{v}{r_{h}^{6}}+\left(r_{h}^{2}+1\right)^{5}-1$

respectively. The specific heat [12]

$C_{Q}=\left(\frac{\partial M}{\partial T_{H}}\right)_{Q}$,

reads as

$C_{Q}=-20 \frac{\pi r_{h}\left(1+r_{h}^{2}\right)^{5}\left[r_{h}^{4}\left(\mu-5 r_{h}^{4}\left(1+r_{h}^{2}\right)^{4}\right)+3 v\right]}{r_{h}^{2}\left\{3 \mu r_{h}^{2}+45 v+r_{h}^{4}\left[11 \mu+5 r_{h}^{2}\left(1+r_{h}^{2}\right)^{5}\right]\right\}+21 v}$.

We observe that absence of root(s) of the denominator implies that the CSBH does not experience phase changes.

2.3.2. For $k \neq \frac{d-1}{4}$ with negative branch

By a similar setting as in the previous subsection, after choosing the negative branch of the solution one gets

$f_{\text {odd }}(r)=1-r^{2}+\left(1+M+\frac{\mu}{r^{2}}+\frac{v}{r^{6}}\right)^{\frac{1}{5}}$. 
We note that the integration constant $M$ and the parameters $\mu$, $v$ have the same values as in Eq. (45). In this branch it is readily seen that there is no restriction on $M$, since the expression in the parenthesis is always positive. In this case also we use the same definitions to find

$T_{H}=\frac{1}{4 \pi} f^{\prime}\left(r_{h}\right)=-\frac{r_{h}}{2 \pi}-\frac{\mu}{10 \pi r_{h}^{3}\left(1+r_{h}^{2}\right)^{4}}-\frac{3 v}{10 \pi r_{h}^{7}\left(1+r_{h}^{2}\right)^{4}}$,

and

$M=\frac{\mu}{r_{h}^{2}}+\frac{v}{r_{h}^{6}}+\left(r_{h}^{2}-1\right)^{5}-1$,

$C_{Q}=20 \frac{\pi r_{h}\left(r_{h}^{2}-1\right)^{5}\left[r_{h}^{4}\left(\mu-5 r_{h}^{4}\left(r_{h}^{2}-1\right)^{4}\right)+3 v\right]}{r_{h}^{2}\left\{-3 \mu r_{h}^{2}+45 v+r_{h}^{4}\left[11 \mu+5 r_{h}^{2}\left(r_{h}^{2}-1\right)^{5}\right]\right\}-21 v}$.

The zeros of the denominator implies possible phase changes in the CSBH, however, the fact that $T_{H}<0$ makes this particular case questionable.

\section{Conclusion}

With the exception of highly symmetric cases finding general integrals to Einstein's field equations in general relativity remained ever challenging. Add to that the most general Lovelock gravity and YM hierarchies, doubtless makes it further challenging. By resorting to a previously known theorem in generating solutions and simplicity of power-YM theory/hierarchy aided in obtaining such particular integrals. The reported static, spherically symmetric metrics are valid in all higher dimensions and occurrence of polynomials with rational powers in closed form seems to be their characteristic feature. A particular example refers to the 11dimensional Chern-Simons (CS) gravity in which the intricacy of the metric function is clearly seen. Determination of zeros of such a function remains a mathematical challenge. For particular dimensions, i.e. $d=8,9$, we plot in Figs. 1 and 2 explicit formation of horizons. From the thermodynamical analysis we evaluate the relevant quantities and investigate the possibility of phase transitions in this model. One particular example that yields $T_{H}<0$, must be discarded as non-physical. The causality and energy conditions discussed in Appendix A guide us to fix the acceptable dimensions for each particular case.

\section{Acknowledgements}

We are indebted to the anonymous referee for drawing our attention to some erroneous statements in the original version of the Letter.

\section{Appendix A. Energy conditions}

When a matter field couples to any system, energy conditions must be satisfied for physically acceptable solutions. We follow the steps as given in [9].

\section{A.1. Weak energy condition (WEC)}

$$
\begin{aligned}
& T^{\mu}{ }_{v}=-\frac{1}{2} \sum_{k=1}^{q} b_{k} \mathcal{F}^{k} \operatorname{diag}[1,1, \xi, \xi, \ldots, \xi], \text { and } \\
& \xi=\left(1-\frac{4 k}{d-2}\right) .
\end{aligned}
$$

The WEC states that,

$\rho \geqslant 0$ and $\rho+p_{i} \geqslant 0 \quad(i=1,2, \ldots, d-1)$

in which $\rho$ is the energy density and $p_{i}$ are the principal pressures given by

$\rho=-T_{t}^{t}=-T_{r}^{r}=\frac{1}{2} \sum_{k} b_{k} \mathcal{F}^{k}, \quad p_{i}=T_{i}^{i}$ (no sum).

The WEC imposes the following conditions on the constant parameters $b_{k}$ and $k$ :

$0 \leqslant b_{k}$ and $0 \leqslant k$.

\section{A.2. Strong energy condition (SEC)}

This condition states that:

$\rho+\sum_{i=1}^{d-1} p_{i} \geqslant 0$ and $\rho+p_{i} \geqslant 0$.

This condition together with the WEC constrain the parameters as

$0 \leqslant b_{k}$ and $\frac{d-2}{4} \leqslant k$.

\section{A.3. Dominant energy condition (DEC)}

In accordance with DEC, the effective pressure $p_{\text {eff }}$ should not be negative i.e. $p_{\text {eff }} \geqslant 0$ where

$p_{\text {eff }}=\frac{1}{d-1} \sum_{i=1}^{d-1} T_{i}^{i}$

One can show that DEC, together with SEC and WEC impose the following conditions on the parameters

$0 \leqslant b_{k}$ and $\frac{d-1}{4} \leqslant k$.

\section{A.4. Causality condition (CC)}

In addition to the energy conditions one can impose the causality condition (CC)

$0 \leqslant \frac{p_{e f f}}{\rho}<1$

which implies

$0 \leqslant b_{k}$ and $\frac{d-1}{4} \leqslant k \leqslant \frac{d-1}{2}$.

\section{References}

[1] D. Lovelock, J. Math. Phys. 12 (1971) 498; D.G. Boulware, S. Deser, Phys. Rev. Lett. 55 (1985) 2656; J.T. Wheeler, Nucl. Phys. B 268 (1986) 737; J.T. Wheeler, Nucl. Phys. B 273 (1986) 732;

M. Banados, C. Teitelboim, J. Zanelli, Phys. Rev. D 49 (1994) 975; J. Crisostomo, R. Troncoso, J. Zanelli, Phys. Rev. D 62 (2000) 084013; R. Aros, R. Troncoso, J. Zanelli, Phys. Rev. D 63 (2001) 084015; R.G. Cai, Phys. Lett. B 582 (2004) 237; R.G. Cai, K.S. Soh, Phys. Rev. D 59 (1999) 044013; R.G. Cai, L.M. Caob, N. Ohta, Phys. Rev. D 81 (2010) 024018; R.G. Cai, Phys. Rev. D 65 (2002) 084014; R.G. Cai, Q. Guo, Phys. Rev. D 69 (2004) 104025; R.C. Myers, J.Z. Simon, Phys. Rev. D 38 (1988) 2434; H.C. Kim, R.G. Cai, Phys. Rev. D 77 (2008) 024045; 
Y. Bri-haye, E. Radu, Phys. Lett. B 661 (2008) 167;

S. Alexeyev, N. Popov, M. Startseva, A. Barrau, J. Grain, J. Exp. Theor. Phys. 106 (2008) 709;

A. Anabalon, N. Deruelle, Y. Morisawa, J. Oliva, M. Sasaki, D. Tempo, R. Troncoso, Class. Quantum Grav. 26 (2009) 065002;

C. Garraffo, G. Giribet, Mod. Phys. Lett. A 23 (2008) 1801;

S.H. Hendi, M.H. Dehghani, Phys. Lett. B 666 (2008) 116;

M.H. Dehghani, S.H. Hendi, Phys. Rev. D 73 (2006) 084021;

M.H. Dehghani, N. Alinejadi, S.H. Hendi, Phys. Rev. D 77 (2008) 104025;

M.H. Dehghani, N. Bostani, S.H. Hendi, Phys. Rev. D 78 (2008) 064031.

[2] H. Maeda, M. Hassaïne, C. Martínez, Phys. Rev. D 79 (2009) 044012;

M. Hassaïne, C. Martínez, Class. Quantum Grav. 25 (2008) 195023;

M. Hassaïne, C. Martínez, Phys. Rev. D 75 (2007) 027502;

S.H. Hendi, B.E. Panah, Phys. Lett. B 684 (2010) 77;

S.H. Hendi, H.R. Rastegar-Sedehi, Gen. Rel. Grav. 41 (2009) 1355;

S.H. Hendi, Phys. Lett. B 678 (2009) 438;

S.H. Hendi, Class. Quantum Grav. 26 (2009) 225014;

S.H. Hendi, B. Eslam Panah, Phys. Lett. B 684 (2010) 77;

S.H. Hendi, Phys. Lett. B 690 (2010) 220;

H.A. González, M. Hassaïne, C. Martínez, Phys. Rev. D 80 (2009) 104008.

[3] S.H. Mazharimousavi, M. Halilsoy, Phys. Lett. B 681 (2009) 190.

[4] D.H. Tchrakian, Phys. Lett. B 150 (1985) 360.

[5] D.H. Tchrakian, in: C.N. Yang, M.L. Ge, X.W. Zhou (Eds.), Differential Geometric Methods in Theoretical Physics, Int. J. Mod. Phys. A (Proc. Suppl.) 3A (1993) 584 ;
G.M. O’Brien, D.H. Tchrakian, J. Math. Phys. 29 (1988) 1212;

S.H. Hendi, H.R. Rastegar-Sedehi, Gen. Rel. Grav. 41 (2009) 1355;

E. Radu, C. Stelea, D.H. Tchrakian, Phys. Rev. D 73 (2006) 084015;

P. Breitenlohner, D. Maison, D.H. Tchrakian, Class. Quantum Grav. 22 (2005) 5201;

P. Breitenlohner, D.H. Tchrakian, Class. Quantum Grav. 26 (2009) 145008;

E. Radu, D.H. Tchrakian, Y. Yang, Phys. Rev. D 77 (2008) 044017.

[6] S.H. Mazharimousavi, M. Halilsoy, Phys. Rev. D 76 (2007) 087501.

[7] S.H. Mazharimousavi, O. Gurtug, M. Halilsoy, Int. J. Mod. Phys. D 18 (2009) 2061;

S.H. Mazharimousavi, O. Gurtug, M. Halilsoy, Class. Quantum Grav. 27 (2010) 205022, arXiv:0911.1919.

[8] T.T. Wu, C.N. Yang, in: H. Mark, S. Fernbach (Eds.), Properties of Matter Under Unusual Conditions, Interscience, New York, 1969, p. 349;

P.B. Yasskin, Phys. Rev. D 12 (1975) 2212;

S.H. Mazharimousavi, M. Halilsoy, Phys. Lett. B 659 (2008) 471.

[9] S.H. Mazharimousavi, M. Halilsoy, Z. Amirabi, Gen. Rel. Grav. 42 (2009) 261.

[10] F. Izaurieta, E. Rodriguez, P. Salgado, Phys. Lett. B 586 (2004) 397; S. Willison, Phys. Rev. D 80 (2009) 064018;

M. Bañados, C. Teitelboim, J. Zanelli, Phys. Rev. D 49 (1994) 975 ;

R.C. Myers, J.Z. Simon, Phys. Rev. D 38 (1988) 2434;

G. Allemandi, M. Francaviglia, M. Raiteri, Class. Quantum Grav. 20 (2003) 5103; G.A.S. Dias, S. Gao, J.P.S. Lemos, Phys. Rev. D 75 (2007) 024030.

[11] M. Bañados, C. Teitelboim, J. Zanelli, Phys. Rev. D 49 (1994) 975.

[12] J. Crisóstomo, R. Troncoso, J. Zanelli, Phys. Rev. D 62 (2000) 084013. 